\title{
Ethanol in cell culture: disinfectant or contaminant?
}

\section{Opinion}

Cell experiment is one of the potential tools of scientific research. It represents an initial step of vital importance for further in vivo and clinical investigations since it saves time and gives an initial estimation about underlying mechanisms of pathophysiological cases under study. Most of the laboratories all over the world include at least one cell culture room, which means that having a command of skills to work on cells is central for a scientist. Before conducting a clinical study, a researcher starts with primary steps like animal experiments (mouse, non-human primates, etc.), but preferably beginning with cell experiments since cells are the elementary building unit of living organisms and working on cells give quick results for further studies and is less costly.

A huge number of published papers have been reporting interesting results obtained through working on human or animal cells in different science fields and almost all diseases for which pathophysiological mechanisms are under study, such as neurodegenerative diseases, ${ }^{1,2}$ and diabetes. ${ }^{3}$

Hence the importance of paying attention to all the steps in a cell experiment since the following experiments are based on its results. Herein, I am writing this paper to draw attention to an important point in this regard, in order to guarantee obtaining accurate findings without any invisible or underestimated influencing factors, and I focus on the effects of ethanol in this report.

Ethanol solution is used widely as a disinfectant in cell culture room at two usual percentages, which are $70 \%$ and $75 \%$. The experimenter often sprays it on experimental tools, on gloves, lab coat, and over other necessary experiment items. Here we want to put a spotlight on the importance of being careful on the quantity sprayed and where to spray it, and particularly to avoid its contact with experimental cells, since this will lead to radical influence on cells pathophysiological condition. For instance, ethanol can induce neurodegenerative diseases, ${ }^{4}$ and it interferes with pathological mechanisms of diabetes. ${ }^{5}$

In conclusion, ethanol is largely utilized as antiseptic in cell experiment environment, and at the same time it has a huge number of possible implications in different cellular mechanisms, and this proves the importance of reasonable use of its solution. In addition, it can dissolve many experimental compounds, and also pen ink used for writing notes on petri dishes, microplates, cryogenic tubes, etc. and this may cause much risk of cell contamination with undesirable factors. Hence the necessity of being careful when spraying this useful disinfectant, but also harmful product when ndesirable if being in contact with cells when studying different diseases mechanisms, particularly those for which ethanol may play a pivotal role. Moreover, when this alcohol is being investigated for its role in certain
Volume 2 Issue 4 - 2015

\author{
Besma Boubertakh ${ }^{1,2}$ \\ 'Key Laboratory of Animal Models and Human Disease \\ Mechanisms of Chinese Academy of Sciences \& Yunnan Province, \\ Kunming Institute of Zoology, China \\ ${ }^{2}$ University of Chinese Academy of Sciences, China
}

Correspondence: Besma Boubertakh, Key Laboratory of Animal Models and Human Disease Mechanisms, Kunming Institute of Zoology Chinese Academy of Sciences, No. 32 liaochang Donglu, Kunming 650223, China, Email:www.boubertakhbesma@outlook.com

Received: September 19,2015 | Published: September 23 2015

pathologies, any extra amounts of it due to careless of experimenter would influence seriously experiment results. That is why, I am writing this report to draw experimenter attention to this essential point for more accurate and effective results for cell experiments.

\section{Acknowledgments}

None.

\section{Conflicts of interest}

The author declares that there is no conflict of interest.

\section{References}

1. Mairuae N, Connor JR, Lee SY, et al. The effects of okra (Abelmoschus esculentus Linn.) on the cellular events associated with Alzheimer's disease in a stably expressed HFE neuroblastoma SH-SY5Y cell line. Neurosci Lett. 2015;603:6-11.

2. Rinaldi DE, Corradi GR, Cuesta LM, et al. The Parkinson-associated human P5B-ATPase ATP13A2 protects against the iron-induced cytotoxicity. Biochim Biophys Acta. 2015;1848(8):1646-1655.

3. Verma S, Chandra H, Banerjee M. Cyclooxygenase 1 (COX1) expression in Type 2 diabetes mellitus: A preliminary study from north India. Egyptian Journal of Medical Human Genetics. 2016;17(1):41-45.

4. Yang JY, Xue X, Tian H, et al. Role of microglia in ethanol-induced neurodegenerative disease: Pathological and behavioral dysfunction at different developmental stages. Pharmacol Ther. 2014;144(3):321-337.

5. Gao Q, Wang HJ, Wang XM, et al. Activation of ALDH2 with ethanol attenuates diabetes induced myocardial injury in rats. Food Chem Toxicol. 2013;56:419-424. 